This is the final peer-reviewed accepted manuscript of:

Gamberini, M., Passarelli, L., Bakola, S., Impieri, D., Fattori, P., Rosa, M.G.P. and Galletti, C. (2017), Claustral afferents of superior parietal areas PEC and PE in the macaque. J. Comp. Neurol., 525: 1475-1488.

The final published version is available online at: https://doi.org/10.1002/cne.24052

Rights / License:

The terms and conditions for the reuse of this version of the manuscript are specified in the publishing policy. For all terms of use and more information see the publisher's website.

This item was downloaded from IRIS Università di Bologna (https://cris.unibo.it/)

When citing, please refer to the published version. 


\title{
Claustral Afferents of Superior Parietal Areas PEc and $P E$ in the Macaque
}

\author{
Michela Gamberini, ${ }^{1}$ Lauretta Passarelli, ${ }^{1}$ Sophia Bakola, ${ }^{1,2,3}$ Daniele Impieri, ${ }^{1}$ Patrizia Fattori, ${ }^{1}$ \\ Marcello G. P. Rosa, ${ }^{2,3 \dagger}$ and Claudio Galletti ${ }^{1 \dagger *}$ \\ ${ }^{1}$ Department of Pharmacy and Biotechnology, University of Bologna, 40126, Bologna, Italy \\ ${ }^{2}$ Biomedicine Discovery Institute and Department of Physiology, Monash University, Clayton, Victoria, 3800, Australia \\ ${ }^{3}$ Australian Research Council, Centre of Excellence for Integrative Brain Function, Monash University Node, Clayton, Victoria, 3800, \\ Australia
}

\begin{abstract}
The exposed surface of the primate superior parietal cortex includes two cytoarchitectonically defined areas, the PEc and PE. In the present study we describe the distribution of neurons projecting from the claustrum to these areas. Retrograde neuronal tracers were injected by direct visualization of regions of interest, and the location of injection sites was reconstructed relative to cytoarchitectural borders. For comparison, the patterns of claustral label that resulted from injections involving neighboring cytoarchitectonic areas were analyzed. We found that the claustral territories sending projections to areas PE and PEc partially overlapped zones previously shown to form projections to the posterior parietal, somatosensory, visual, and motor cortex. The projection zones to the PE and PEc overlapped extensively, and consisted of multiple patches separated by label-free zones. Most
\end{abstract}

of the labeled neurons were located in the posterior-ventral part of the claustrum. Area PE received additional inputs from a posterior-dorsal part of the claustrum, which has been previously reported to project to the somatosensory cortex, while the PEc receives additional input from an anterior-ventral region of the claustrum, which has been reported to project to the visual association cortex. These observations reflect the known functional properties of the PE and PEc, with the former containing neurons that are predominantly involved in somatosensory processing, and the latter including both somatosensory and visual neurons. The present results suggest that the claustrum projections may help coordinate the activity of an extensive neural circuit involved in sensory and motor processing for movement execution. J. Comp. Neurol. 000:000-000, 2016.

INDEXING TERMS: posterior parietal cortex; connectivity; primate; claustrum; superior parietal lobule; sensorimotor input

The claustrum forms a relatively thin, folded sheet of gray matter, inserted between the striatum and the insular cortex, which is surrounded by white matter (Crick and Koch, 2005). The claustrum has been classically considered as a component of the basal ganglia, but its direct projections to the cortex suggest a very different role. However, there are still relatively few data on which to build detailed hypotheses about its function (for comprehensive reviews, consult Smythies et al., 2012; Baizer et al., 2014; Mathur, 2014). Neuroanatomical studies in New and Old World monkeys have revealed widespread connections between the claustrum and neocortical regions in the frontal, occipital, and temporal lobes, as well as in the parietooccipital and posterior parietal regions, and somatosensory areas (Carman et al., 1964; Druga 1968, 1966; Kemp and Powell, 1970; Chadzypanagiotis and Narkiewicz, 1971; Pearson et al., 1982; Baizer et al., 1993; Tanné-Gariepy et al., 2002; Burman et al., 2011; Reser et al., 2014; Milardi et al., 2015).

*CORRESPONDENCE TO: Claudio Galletti, Department of Pharmacy and Biotechnology, Piazza di Porta S. Donato, 2, 40126 Bologna, Italy. E-mail: claudio.galletti@unibo.it 


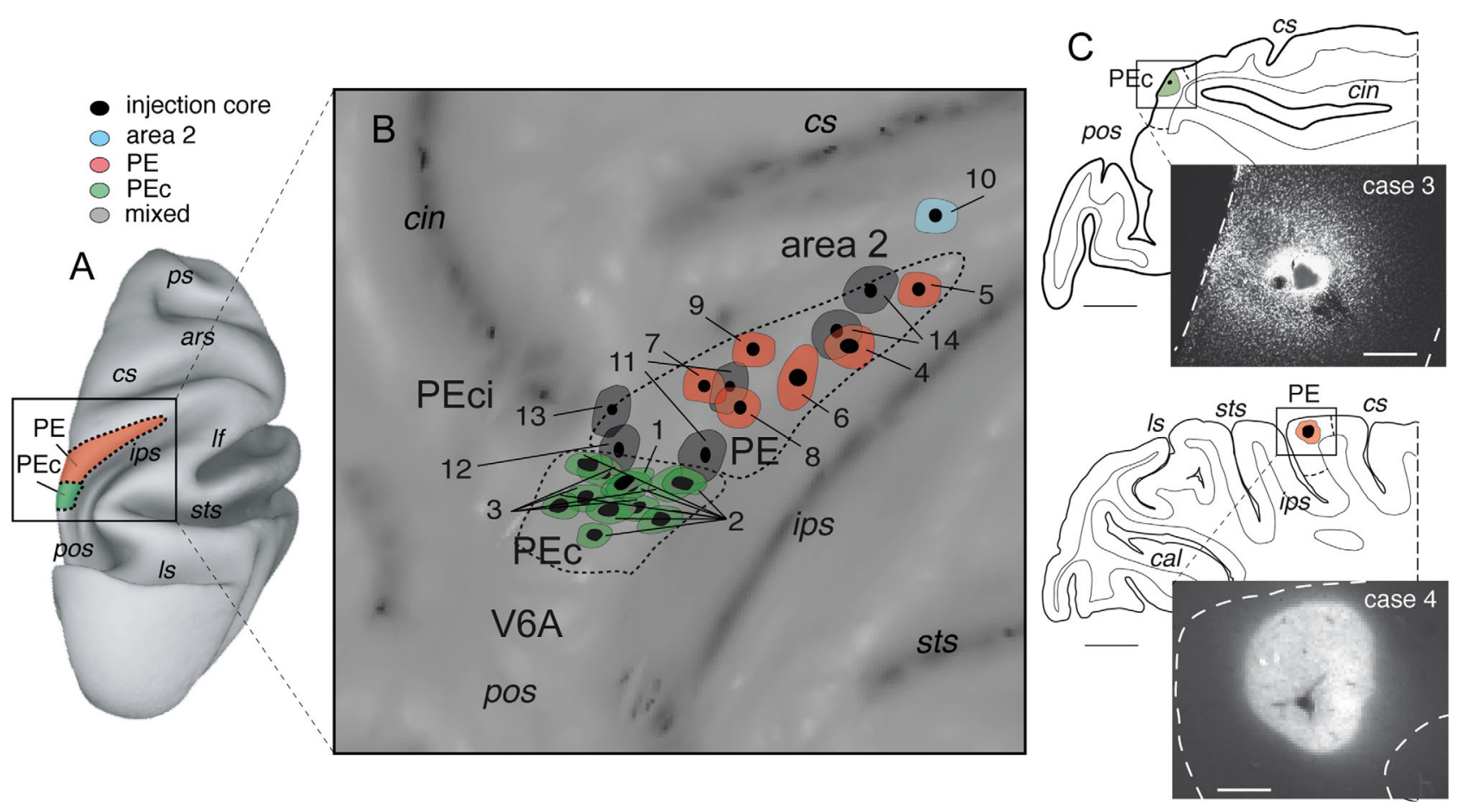

Figure 1. Summary of injection site locations. A,B: Injection sites in six animals are illustrated on a two-dimensional reconstruction (B) of the caudal superior parietal lobe of the right hemisphere of a reference monkey brain shown on the left (A). The dashed contours represent the average cytoarchitectonic borders of the PEC and PE, respectively. C: Examples of injection sites. Parasagittal sections taken at the level of injection sites in case 3 (DY injection in area PEc), and case 4 (CTB-green injection in area PE). Dashed lines within sections indicate the borders of areas PEc or PE. Abbreviations: ars, arcuate sulcus; cal, calcarine fissure; cin, cingulate sulcus; cs, central sulcus; ips, intraparietal sulcus; If, lateral fissure; Is, lunate sulcus; pos, parieto-occipital sulcus; ps, principal sulcus; sts, superior temporal sulcus. Scale bars in $\mathrm{C}=5 \mathrm{~mm}$ for sections and $500 \mu \mathrm{m}$ for injection sites.

Here we investigated the claustral projections to cortical areas PEc and PE located on the exposed cortex of the superior parietal lobule. Area PEc contains visual, somatosensory, and bimodal neurons (Breveglieri et al., $2006,2008)$, most of which are sensitive to the movement and position of hand and eye (Ferraina et al., 2001; Battaglia-Mayer et al., 2001). This area contains an incomplete representation of the body, mainly focused on upper and lower limbs, without an evident topographic organization (Breveglieri et al., 2006, 2008). It has been recently demonstrated that a large percentage of PEc neurons encodes both direction and depth information during arm reaching movements (Hadjidimitrakis et al., 2015), and contributes to hand-target transformations for reaching (Piserchia et al., 2016). In contrast, area PE (which has been traditionally equated to Brodmann's area 5) contains an almost complete representation of the body, with a coarse topographic organization (Taoka et al., 1998, 2000; Padberg et al., 2007). The majority of its neurons respond to proprioceptive stimulation, while fewer cells are activated by tactile stimuli, and even fewer by visual stimuli (Duffy and Burchfiel, 1971; Sakata et al., 1973; Mountcastle et al., 1975).
Area $P E$ is involved in the preparation of limb movements (Burbaud et al., 1991) and in the generation of different types of reference systems for encoding reaching movements (Ferraina and Bianchi, 1994; Lacquaniti et al., 1995; Kalaska, 1996; Batista et al., 1999; Bremner and Andersen, 2012).

Very few studies have investigated the claustrum in macaque monkeys in the context of sensorimotor integration. Neuronal activity recorded in the claustrum while macaques performed arm movements, either visually guided or triggered by memorized information, suggested that claustral neurons could play a role in arm movement execution (Shima et al., 1996). A comparison with neuronal activity in the primary motor cortex showed that neurons of the claustrum, in contrast to those of the motor cortex, showed little selectivity to the type of movement (Shima et al., 1996). Other studies have suggested that the claustrum integrates multisensory information from different sensory cortices (Ettlinger and Wilson, 1990). The present study defines the origin of projections from the claustrum to the PEC and $\mathrm{PE}$, and links these results to previous observations on claustral projections to other nodes of the cortical 
TABLE 1.

Injection Sites and Neuronal Tracers Employed in the Experiments

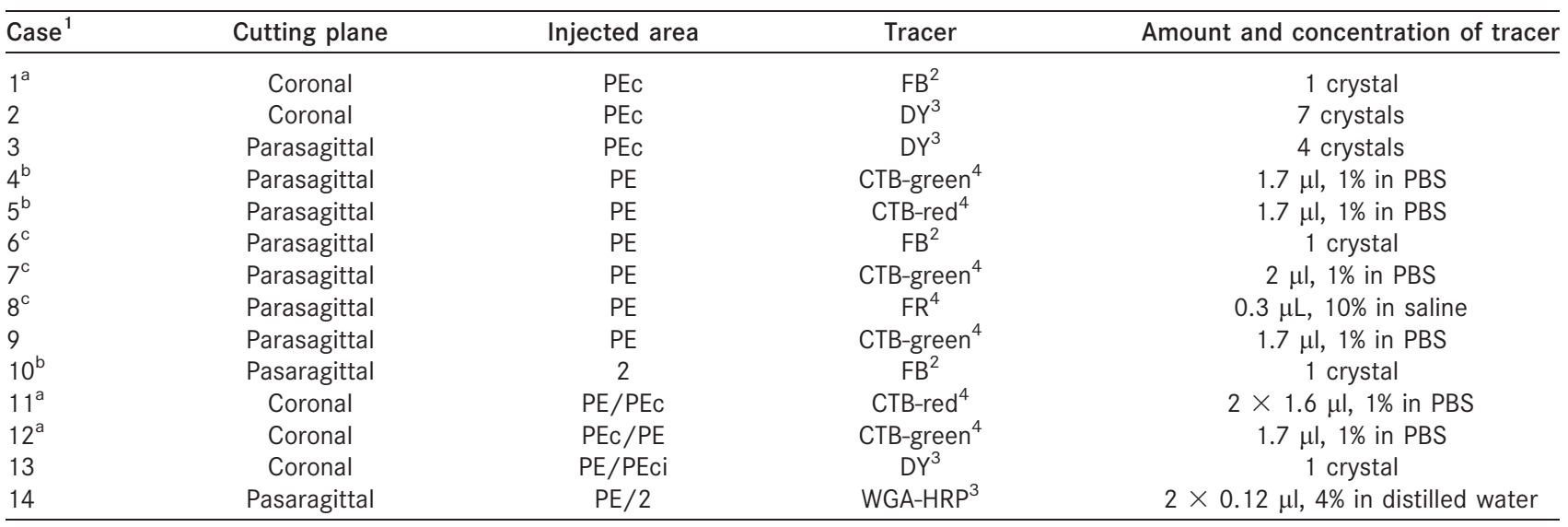

${ }^{1}$ The letters $a, b$, and c correspond to the same hemisphere.

${ }^{2}$ Polysciences Europe, Germany.

${ }^{3}$ Sigma Aldrich.

${ }^{4}$ Molecular Probes.

Abbreviations: CTB, cholera toxin B; DY, Diamidino Yellow; FB, Fast Blue; FR, Fluoro-Ruby; PBS, phosphate-buffered saline; WGA-HRP, wheat germ agglutinin-horseradish peroxidase.

network of areas involved in movement planning and visuomotor integration.

\section{MATERIALS AND METHODS}

Experimental protocols were approved by the Bioethics Committee of the University of Bologna, in accordance with the guidelines of the European Directive 86/609/EEC, and the revised Directive 2010/63/ EU for the Care and Use of Animals for Scientific Purposes. In total, 14 retrograde tracer injections were placed in eight hemispheres of six male adult monkeys (Macaca fascicularis, 3-7 kg). The injections were aimed at areas PEc and PE, located in the superior parietal lobule (Fig. 1A), based on sulcal morphology. The attribution of each injection site to specific areas was based on postmortem analysis of cyto- and myeloarchitectural material following previously defined criteria (Bakola et al., 2010, 2013; Luppino et al., 2005).

Figure $1 \mathrm{~B}$ illustrates the extent and location of the injection sites relative to histological boundaries of cortical areas, projected onto a flat map reconstruction of a reference macaque brain obtained with the software CARET (Van Essen et al., 2001). For each injection, Figure $1 \mathrm{~B}$ and $\mathrm{C}$ shows both the core of the injection (black spot) and the halo zone (colored region around the core). Three of the injections were within the limits of area PEc, and six were within area PE. In other cases, as shown in Figure $1 \mathrm{~B}$, the injection sites crossed the boundary between the PE and PEc (two injections), or the boundary between one of these and an adjacent area (two injections). Finally, one of the injection sites was entirely confined within rostral parie- tal area 2. Table 1 presents details of individual injections.

Full details of the surgical procedures have been described previously (Bakola et al., 2010, 2013; Galletti et al., 2001). Briefly, in all animals the target region was visualized during surgery under aseptic conditions. The animals were pretreated with atropine $(0.05 \mathrm{mg} / \mathrm{kg}$, i.m.) and anesthetized with ketamine hydrochloride $(12 \mathrm{mg} /$ $\mathrm{kg}$, i.m.) followed, after 30 minutes, with sodium thiopental (8 mg/kg, i.v. with supplemental doses as required). To avoid edema, mannitol was administered intravenously ( $1 \mathrm{~g} / \mathrm{kg}$ ). The animals were secured to a stereotaxic frame and, after craniotomy, the superior parietal lobule was exposed and the dura mater retracted. Neuronal tracers were injected through a Hamilton microsyringe that had been fitted with a glass micropipette attached to the needle. The tracers Fast Blue (FB) and Diamidino Yellow (DY) were directly applied as crystals by visual inspection of the exposed cortex (Rosa and Tweedale, 2005). At the end of the surgery, the exposed cortex was covered with surgical foam. The bone was replaced, and the dura mater and the wound were sutured. Analgesics (Ketorolac, $1 \mathrm{mg} / \mathrm{kg}$, i.m., for 2-3 consecutive days) and antibiotics (erythromycin, 1-1.5 $\mathrm{ml} / 10 \mathrm{~kg}$ ) were administered postoperatively. In all cases, the veterinary staff of the University of Bologna monitored physiological parameters during surgery, as well as the animal's recovery in subsequent days.

\section{Histological procedures}

After a variable survival period (14 days for fluorescent tracers, and 2 days for wheat germ agglutinin- 


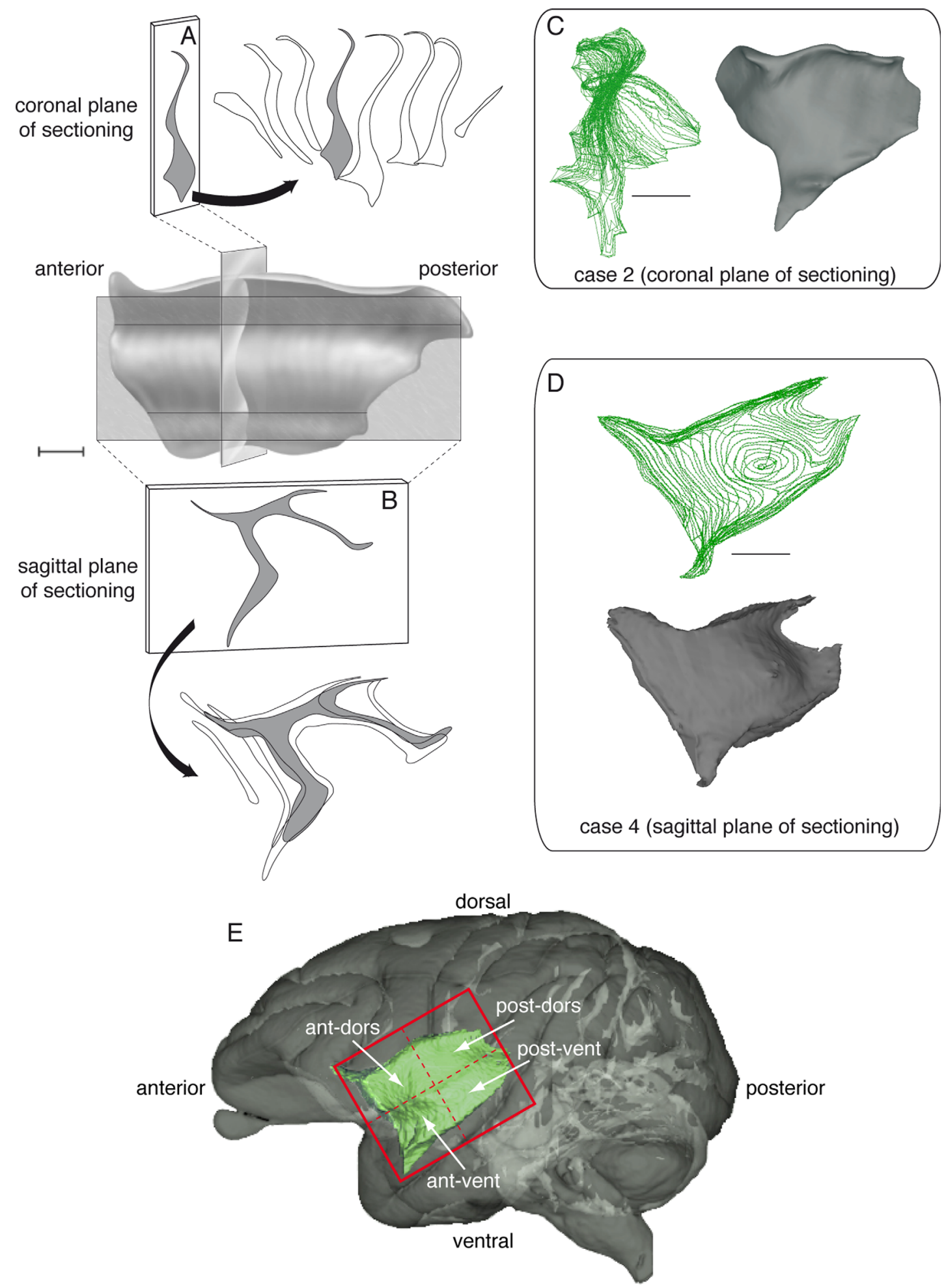

Figure 2. Anatomy of the claustrum. A,B: Illustration of the sheet-like structure of the claustrum of a young squirrel monkey (modified from Fig. 1 of Crick and Koch, 2005). The insets show single coronal (A) and sagittal (B) sections. C,D: Green outlines: claustrum contours in a case sectioned in the coronal plane and another sectioned in the parasagittal plane, respectively. The gray volumes represent 3D reconstructions of the lateral surface of the claustrum, prepared using the software CARET. E: Lateral view of the left hemisphere of a macaque brain showing (in green) the approximate anatomical location and shape of the claustrum (from Brainlnfo: http://braininfo.rprc. washington.edu/TemplateNeuroMaps.aspx). The red rectangle illustrates the anatomical quadrants used for the present analysis. Abbreviations: ant-dors: anterior-dorsal; ant-vent: anterior-ventral; post-dors: posterior-dorsal; post-vent: posterior-ventral. Scale bar $=1.2 \mathrm{~mm}$ in A; $5 \mathrm{~mm}$ in $\mathrm{C}, \mathrm{D}$. 


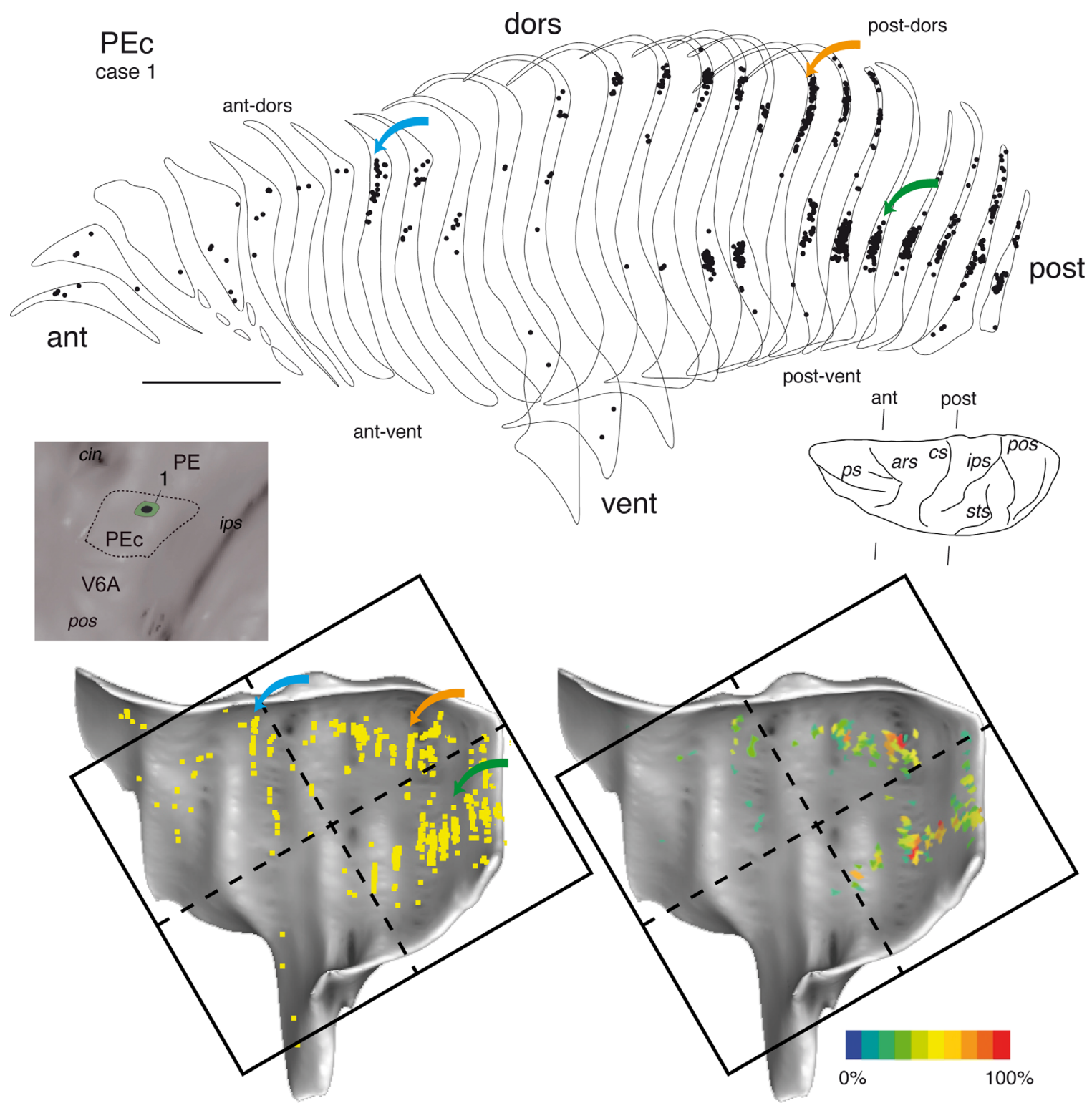

Figure 3. Distribution of retrogradely labeled cells after injection in area PEc (case 1). Top: Outlines of the claustrum in this brain, which was sectioned in the coronal plane. Locations of single-labeled neurons are shown as black circles, and colored arrows point to patches in the sections. Corresponding places are shown in the 3D reconstruction (blue in the ant-dors quadrant, orange in the post-dors quadrant, and green in the post-vent quadrant). The insert on the left shows the location of the injection site. Bottom: Lateral views of 3D reconstructions of the claustrum, illustrating the distribution (left) and density (right) of labeled cells. Color scale indicates the relative density of labeled cells, counted within $300 \times 300 \mu \mathrm{m}$ units, as a percentage of the maximum value. In this and other figures the claustrum is represented with the anterior end at the left, irrespective of the hemisphere injected, to facilitate comparisons. Abbreviations: ant-, anterior; dors-, dorsal; post-, posterior; vent-, ventral. Other details and abbreviations as in Figure 1. Scale bar $=5 \mathrm{~mm}$ at top.

horseradish peroxidase [WGA-HRP]), the animals were treated with ketamine hydrochloride $(15 \mathrm{mg} / \mathrm{kg}$, i.m.). Following loss of consciousness, they received a lethal dose of sodium thiopental (i.v.), and, upon cardiac arrest, were perfused with 3 liters of normal saline solution, followed by 5 liters of $4 \%$ paraformaldehyde in 0.1 $\mathrm{M}$ phosphate buffer at $\mathrm{pH} 7.4(3.5 \%$ in the case of the WGA-HRP injection), and 4 liters of $5 \%$ glycerol in the same buffer. The brains were removed from the skulls, photographed from all views, and cryoprotected by immersion in $0.1 \mathrm{M}$ phosphate buffer solutions containing glycerol ( $10 \%$ and $20 \%$; all cases). The brains were then snap-frozen and stored at $-80^{\circ} \mathrm{C}$. Sections $(60$ $\mu \mathrm{m})$ were obtained using a freezing microtome. In most cases the brain was sectioned in parasagittal plane. The preference for the sagittal plane was dictated by the need to determine the histological boundaries of the PEc, the PE, and area 2, which are best visualized 

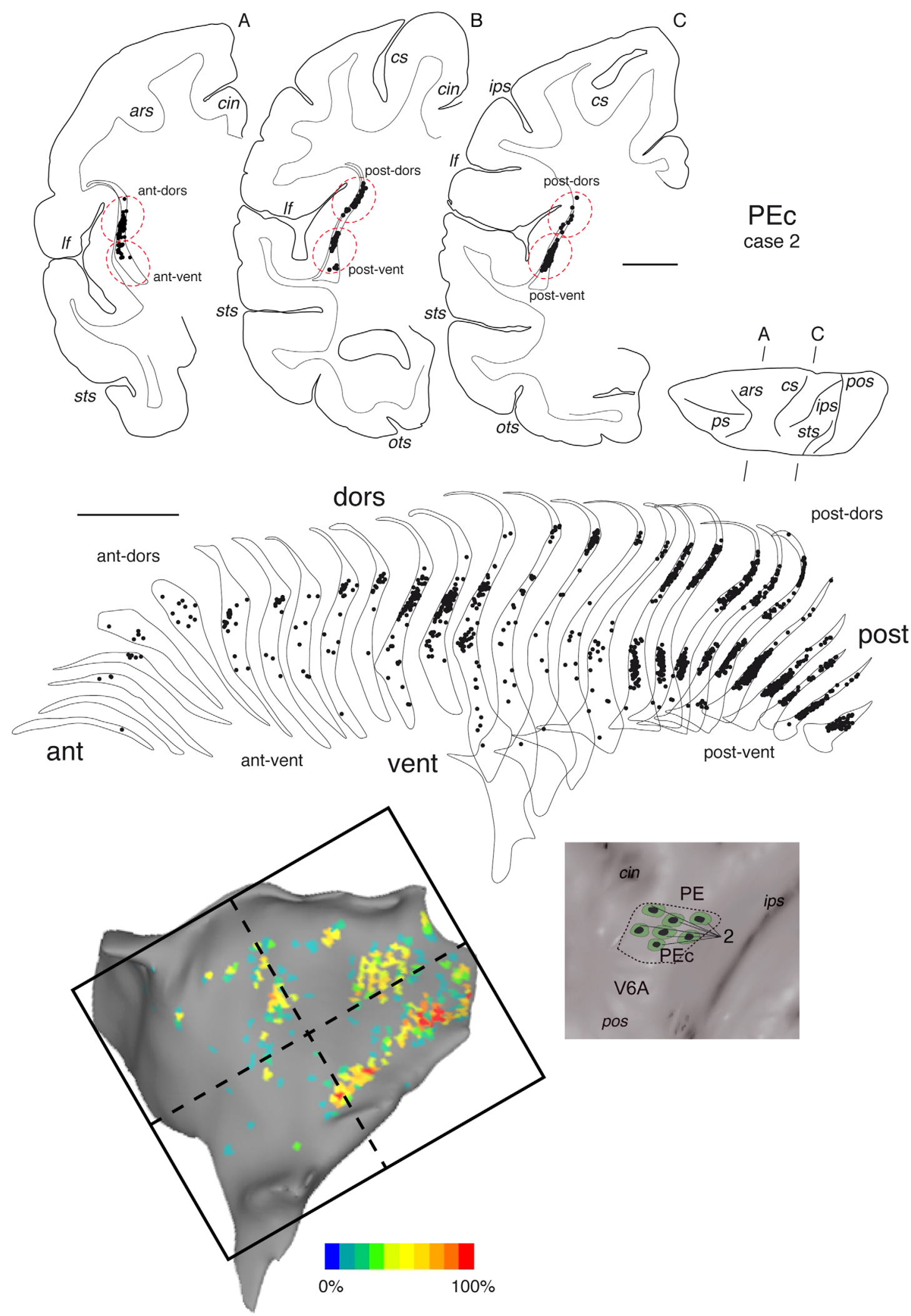

Figure 4. Distribution of retrogradely labeled cells after injection in area PEc (case 2). Top: Coronal sections at the levels indicated on a dorsal view of the brain. Labeled cells are represented as black dots. Dashed red ovals indicate labeled cells attributed to different claustral quadrants. Center: Set of claustrum contours. Bottom: 3D reconstruction illustrating the density of labeled neurons (left) and the location of injection sites (right). Abbreviation: ots, occipitotemporal sulcus. Other details and abbreviations as in Figures 1-3. Scale bar $=5 \mathrm{~mm}$ at top. 


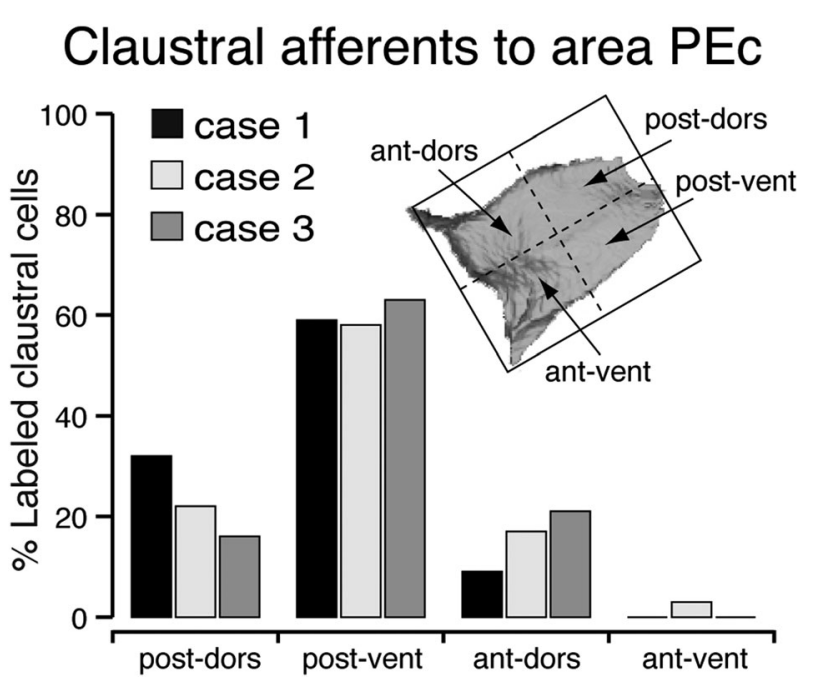

Figure 5. Percentage of labeled cells in the four quadrants of the claustrum after injections confined within the cytoarchitectonic limits of area PEc.

in this plane of section. Five series of sections were obtained, one of which was always stained for Nissl substance and another for myelin (Gallyas, 1979). The other series were left unstained for fluorescence observation, or processed to reveal WGA-HRP using the tetramethylbenzidine method (Mesulam and Rosene, 1979). All sections were coverslipped with DPX after quick steps of dehydration in $100 \%$ ethanol, and cleared with xylene.

\section{Data analysis}

The sections were examined for labeled neurons using a microscope (Zeiss Axioscope) equipped with $10 \times$ and $20 \times$ objectives. In each case, the entire hemisphere ipsilateral to the injection site was examined for retrograde label. Although anterograde label from some of the injections was visualized, only the retrograde label has been quantified for the purposes of the present report. The section outlines and location of labeled neurons were plotted at $600 \mu \mathrm{m}$ intervals, using a computerized system linked to $X / Y$ transducers mounted on the microscope stage.

The histological criteria used for the definition of the boundaries of areas around the injection sites have been fully described in previous studies (Bakola et al., 2010, 2013; Galletti et al., 2001; Luppino et al., 2005). The present report focuses on injections that were found to be confined to a single architectonic area, although data from injections that crossed areal boundaries have been used as comparison and/or confirmation of particular aspects of the data, as detailed in the Results section.
The limits of the claustrum were plotted together with the external (pial) and internal (gray/white matter boundaries) contours of each histological section stained with the Nissl protocol at $300 \mu \mathrm{m}$ intervals. To define the labeled region of the claustrum, a camera lucida attachment was used to bring stained histological sections into register with the corresponding drawings.

Figure 2 shows examples of 3D reconstructions of the claustrum in cases cut in coronal ( $A$ and $C$ ) or sagittal $(B$ and $D)$ planes. The $3 D$ reconstructions of the claustrum shown in Figures $2 \mathrm{C}$ and $\mathrm{D}$ were obtained from section contours with CARET software (Van Essen et al., 2001), according to the procedures described previously (Galletti et al., 2005; Gamberini et al., 2009). We used the midthickness contours of the cortical gray matter to align brain sections, to reconstruct the brain shape in each case. Adjustments were applied to the contours of the claustrum only in specific cases, to improve local alignment. CARET tools allowed us to display individual labeled neurons in the claustrum reconstructions (Galletti et al., 2005), or to prepare labeled neuron density maps (Bakola et al., 2010; Passarelli et al., 2011) in $300 \times 300 \mu \mathrm{m}$ area units superimposed on a lateral view of the claustrum. The area unit that contained the highest number of labeled neurons was considered as reference, and the density of neurons was expressed as a percentage of this maximum unit value (Rosa et al., 2009). To facilitate the comparison between cases, claustral representations will always be represented as the left hemisphere observed from the lateral surface (Fig. 2E).

For regional analysis of the location of labeled cells, we followed a subdivision similar to that proposed by Pearson and colleagues (1982). The shape of the reconstructed claustrum was fitted into a rectangle tilted $30^{\circ}$ counterclockwise from horizontal when aligned according to stereotaxic coordinates (see red rectangle in Fig. $2 \mathrm{E})$. This rectangle was subdivided into four quadrants of equal size (dashed red lines in Fig. 2E). The exact aspect ratio of the rectangle was adjusted according to the shape of the reconstructed claustrum in different cases. Analysis of the location of labeled neurons was then performed with reference to the posterior-ventral (post-vent), posterior-dorsal (post-dors), anterior-ventral (ant-vent), and anterior-dorsal (ant-dors) quadrants.

\section{RESULTS}

Here we report the results of tracer injections in areas PEc and PE in eight hemispheres of six animals, together with one injection in area 2 (details in Table 1). Data from an injection that involved both area PE 


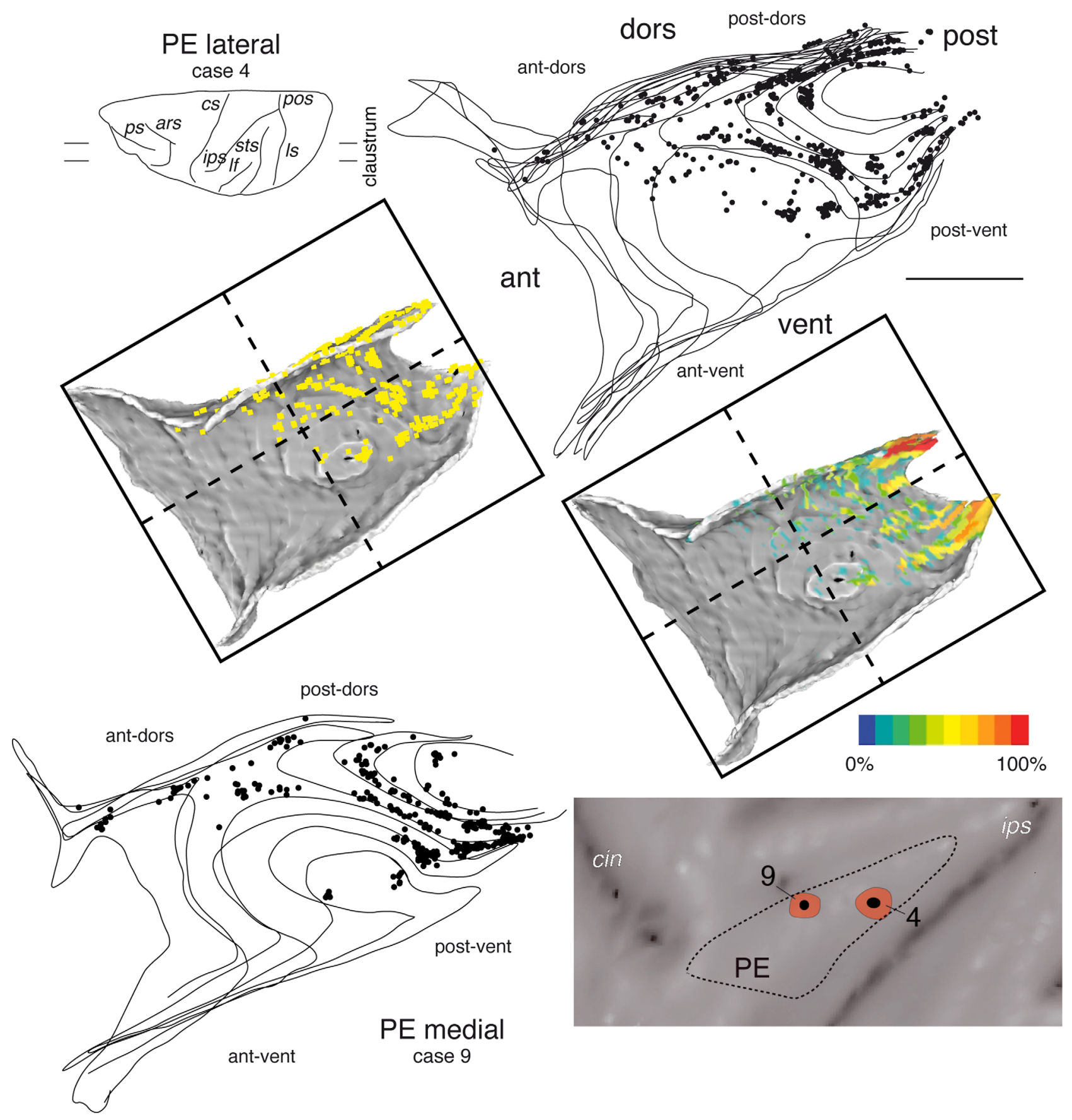

Figure 6. Distribution of retrogradely labeled cells after two injections in area PE (two cases within the cytoarchitectonic limits of area PE). Top: Case 4. Bottom left: Case 9. For both cases a set of superimposed claustrum contours in parasagittal sections is illustrated, as well as the locations of labeled neurons (black circles). Center: 3D reconstructions of the distribution and density of labeled cells in the claustrum. Bottom right: The location of the injection sites. Other details and abbreviations as in Figures $1-3$. Scale bar $=5 \mathrm{~mm}$ at top right.

and area 2 will be discussed only briefly. In all cases, we found that neurons in the claustrum represented only a small fraction of the total number of those labeled by the tracer across the brain (PEc, mean = $5.0 \pm 3.3 \%$; PE, mean $=4.1 \pm 4.1 \%$; area $2,6.2 \%$.

\section{Claustral afferents of areas PEc and PE}

Figures 3 and 4 show two cases of claustral projections to area PEc. In case 1 (Fig. 3), the retrograde tracer FB was injected at a single site, in the anterior part of the PEc (see inset at the left part of Fig. 3, case 
1). In case 2 (Fig. 4), the tracer DY was injected in multiple sites, which collectively encompassed nearly the entire extent of area PEc (see inset at the bottom right part of Fig. 4). Despite the difference in the extent of injection sites, the distribution of labeled neurons in the claustrum was similar in both cases.

In case 1, visual inspection of coronal sections (Fig. 3 , top) revealed that the majority of label in the claustrum formed three patches, two located posteriorly (in the ventral and dorsal parts of the claustrum), and one at an anterior-dorsal location. The 3D reconstruction and density map, generated by the software CARET (Fig. 3, bottom), confirmed the wide distribution of labeled cells, mainly in the posterior half of the claustrum. In case 2 (Fig. 4) the same three patches of labeled cells were clearly evident in coronal sections (top), with the densest label being located in the posterior-ventral part of the structure (see density map, Fig. 4 bottom). Data from a third case with one injection in the PEc (not illustrated) reproduced the above findings. Figure 5 summarizes the quantitative analysis of the three cases in which tracer deposits were entirely confined within the cytoarchitectural limits of area PEc.

Figure 6 shows the claustral afferents in two cases with injections within area PE (locations shown in Fig. 6 , bottom right). One injection was in the lateral part of area PE (case 4, Fig. 6, top), and one in the medial part of the area (case 9, Fig. 6, bottom left). Although the topographic organization of projection neurons was similar to that seen in the projection to area PEc, patches were not as clear as in PEc injection cases, perhaps due to the use of parasagittal sections. The distribution of label, in terms of quadrants of the claustrum, was quite similar to that observed after PEc injections (compare Figs. 5 and 7).

Figure $2 \mathrm{~A}$, together with the $3 \mathrm{D}$ reconstructions in Figures 3 and 4 , shows that the typical sheet of gray matter that forms the claustrum curves laterally for few millimeters in the most dorsal part of the structure, to follow the curvature of the dorsal insular cortex. This very dorsal region of the claustrum was free of labeling after PEc injections (Figs. 3 and 4), but was labeled after $P E$ injections, particularly in its posterior half (Fig. 6).

\section{Claustral afferents of area 2}

One of our injections was placed in area 2. Figure 8 shows the location of labeled neurons in the claustrum after this injection (case 10). The comparison of the patterns of claustrum afferents after area 2 and $P E$ injections is facilitated by the fact that the same hemisphere received one injection in area PE (case 4, Fig. 6) and one injection in area 2 (case 10).

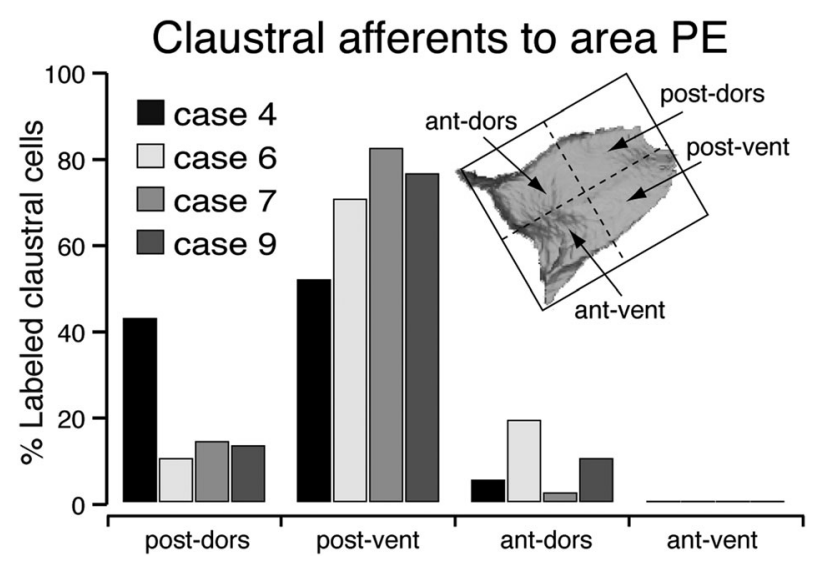

Figure 7. Percentage of labeled cells in the four quadrants of the claustrum after injections confined within the cytoarchitectonic limits of area PE.

The distribution of projection neurons was different from those observed following injections in areas PEc and PE, with concentrations of label in the posteriordorsal and anterior-dorsal quadrants of the claustrum, and fewer labeled neurons in the posterior-ventral quadrant (compare Figs. 7 and 8). Indeed, the dorsal part of the claustrum was fully labeled, in both the posterior and anterior portions of this structure (see reconstruction in the bottom left panels of Fig. 8). The part of the dorsal claustrum that bends laterally to follow the dorsal bank of the lateral fissure was heavily labeled throughout its extent (see parasagittal section shown in Fig. $8 \mathrm{C}$, and the dorsal view of the 3D reconstruction). The strong involvement of this sector of the claustrum in projections to area 2 was confirmed in another case (case 14) in which the injection sites involved both area 2 and area PE (Fig. 9).

\section{DISCUSSION}

The aim of this study was to examine the distribution of claustral afferents to cortical areas of the caudal part of the superior parietal lobule, namely, areas PEc and PE. We found that the common territory of origin of projections to areas PE and PEc includes a large fraction of the claustrum, but essentially spares the anterior-ventral part of this structure. As summarized in Figure 10A, most claustral projections to areas PEc and $\mathrm{PE}$ originate in the posterior-ventral part of the structure $(60-70 \%$ of labeled neurons), followed by the posterior-dorsal ( 20\%), and anterior-dorsal (10-20\%) parts. The posterior part of the claustrum is known to be mainly concerned with sensory information (visual and somatic) (Olson and Graybiel, 1980; Pearson et al., 1982), while the anterior-dorsal part is known to be 

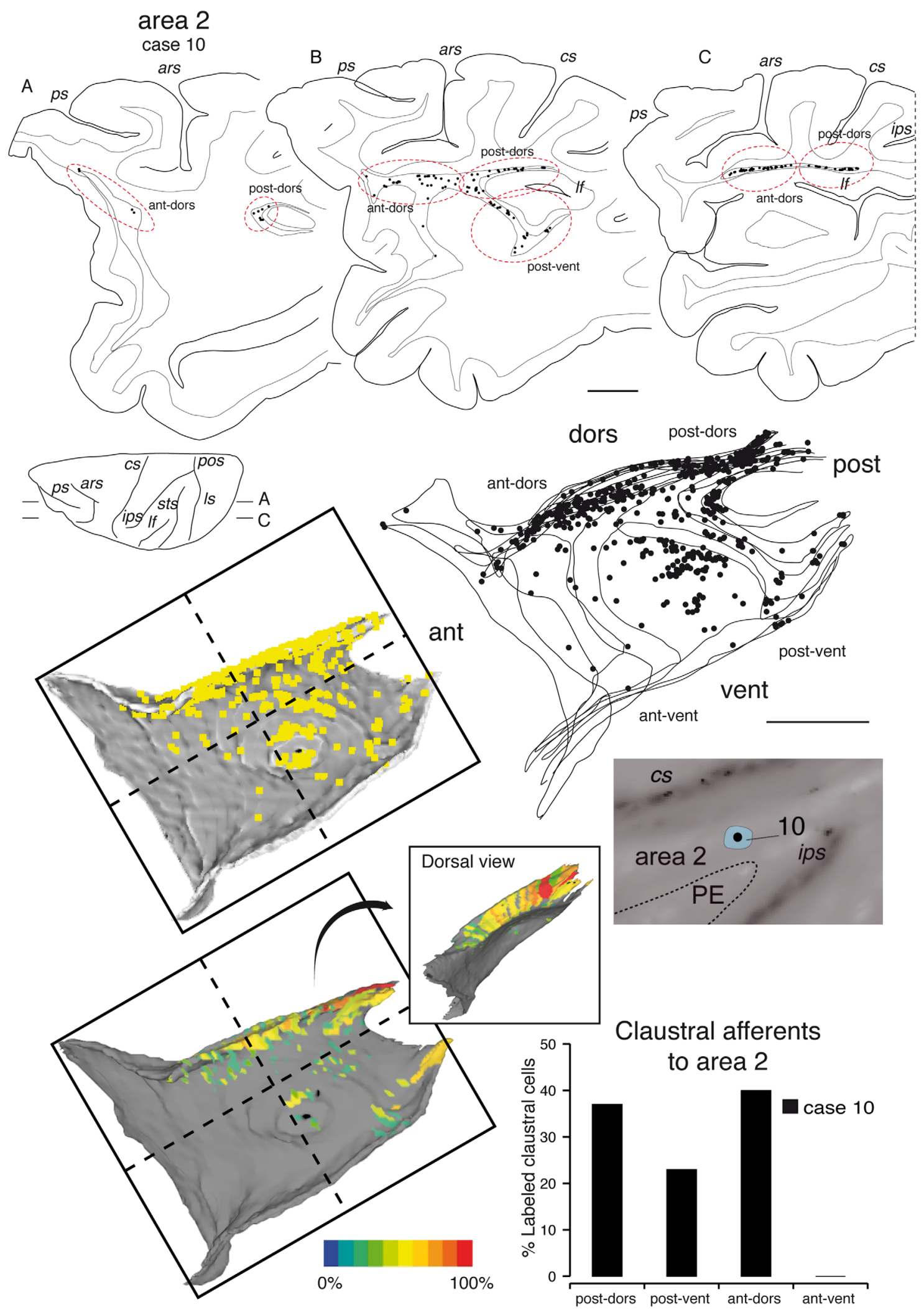

Figure 8. Distribution of retrogradely labeled cells after an injection in area 2 (case 10). Top: Parasagittal sections (A-C) at the levels indicated on a dorsal view of the brain. Center right: Set of claustrum contours with the locations of labeled neurons represented as black dots. Bottom left: 3D reconstruction illustrating the location and density of labeled neurons in lateral view, and dorsal view (insert). Bottom right: The location of the injection site and percentages of labeled neurons in different quadrants of the claustrum. Other details and abbreviations as in Figures 1-4. Scale bars $=5 \mathrm{~mm}$ at top. 


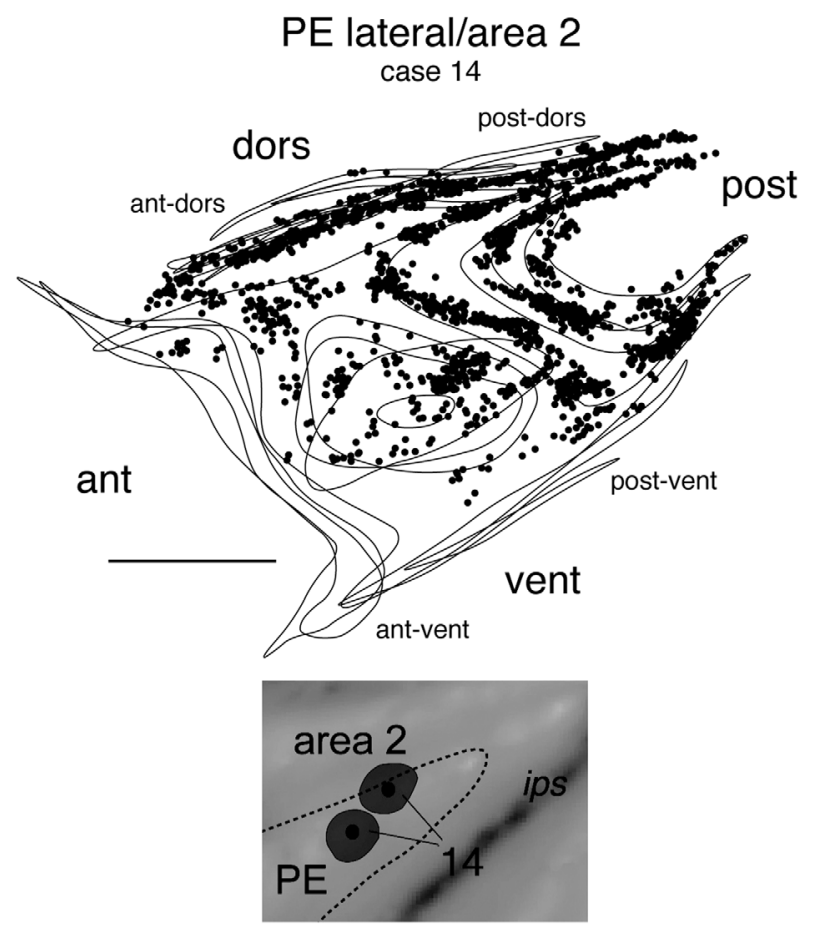

Claustral afferents to PE \& area 2

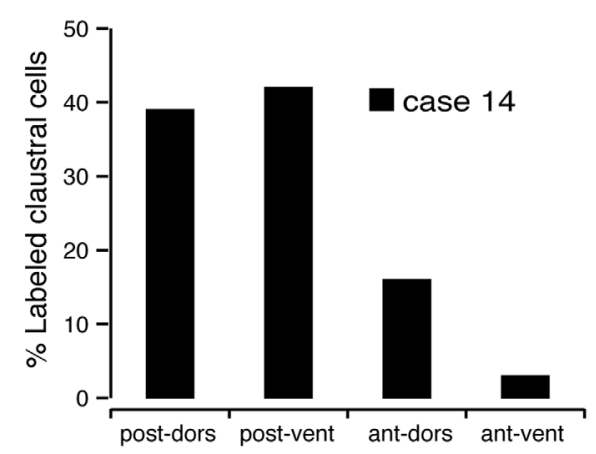

Figure 9. Claustral distribution of retrograde-labeled cells after a case in which tracer was injected at two adjacent sites, which collectively crossed the boundary between area PE and area 2 (case 14). For conventions, see Figure 8 . Scale bar $=5 \mathrm{~mm}$ at top.

connected to the somatomotor cortex (present results; Pearson et al., 1982; Minciacchi et al., 1991; Mathur, 2014). By comparison, the anterior-ventral portion of the claustrum, which is not connected with any of the areas considered in the present study, has been reported to form strong connections with the granular prefrontal cortex (Reser et al., 2013).

The considerable overlap of claustral labeling after injections in different parietal areas (PEc, PE, area 2) is not surprising. Pearson and colleagues (1982) reported that a similar degree of overlap may be found after injections of two widely separated, but interconnected areas, referring to cortical areas located in parietal and frontal lobes. Here we show that neighboring cytoarchitectural subdivisions of the parietal lobe, which are strongly and reciprocally interconnected (Bakola et al., 2010, 2013; Pons and Kaas, 1986) show a similar degree of overlap. This supports the idea that one cortical area may influence another, not only through the association cortical fibers, but also through the claustrum (Pearson et al., 1982).

As shown by comparison of panels $C$ and $D$ in Figure 10 , our observations are in good agreement with those of Pearson et al. (1982) in other respects. Neurons forming projections to area 2 (part of the $\mathrm{S} 1$ complex) were concentrated along the entire dorsal limit of the claustrum, while those projecting to areas PE and PEc (parts of area 5; Pandya and Seltzer, 1982) were, on average, shifted caudally and ventrally. Furthermore, the claustral territories projecting to areas PE and PEc appear to partially overlap with those projecting to frontal motor and premotor areas (Tanné-Gariepy et al., 2002). Although the origins of claustral afferents to areas PEC and PE overlapped in the posterior-ventral quadrant of the claustrum, the strongest foci of label appear to occupy somewhat different regions. In particular, the origins of afferent projections to area PEc seem to extend further into the "visual" sector of the claustrum (i.e., regions that have been demonstrated to project to the extrastriate cortex; Maioli et al., 1983; Gattass et al., 2014; Fig. 10E,F), in comparison with those to area PE. These data agree well with a primarily somatosensory nature of area PE and a bimodal visual and somatosensory nature of area PEc (Breveglieri et al., 2006, 2008). On a historical note, based on cytoarchitecture, Brodmann (1909) originally considered the territory currently assigned to area PEc to be part of the area 7 complex, while the current area PE was assigned to area 5. The spatial shift in the origin of claustral projections to the PE and PEc shown in Figure $10 \mathrm{C}$ can be related to that described by Pearson et al. (1982), for the projections to areas 5 and 7, although modern visualization techniques allow a better appreciation of the patchy nature of the projections, and the gradual nature of the spatial shift.

According to Gattass et al. (2014), the claustrum can be subdivided into four sectors according to projections to the occipital, parietal, temporal, and frontal lobes (Fig. 10F, right). Our data do not agree with this summary, because many of the afferents to posterior parietal areas PE and PEc originate from a claustral region that has been assigned to the occipital lobe, rather than the parietal lobe (compare Figs. 10C and F). In fact, one way in which our data extend those of many previous studies is by emphasizing the complexity of the spatial distribution of projection neurons. Rather 


\section{Average percentage of labeled cells to areas PEc-PE-2}
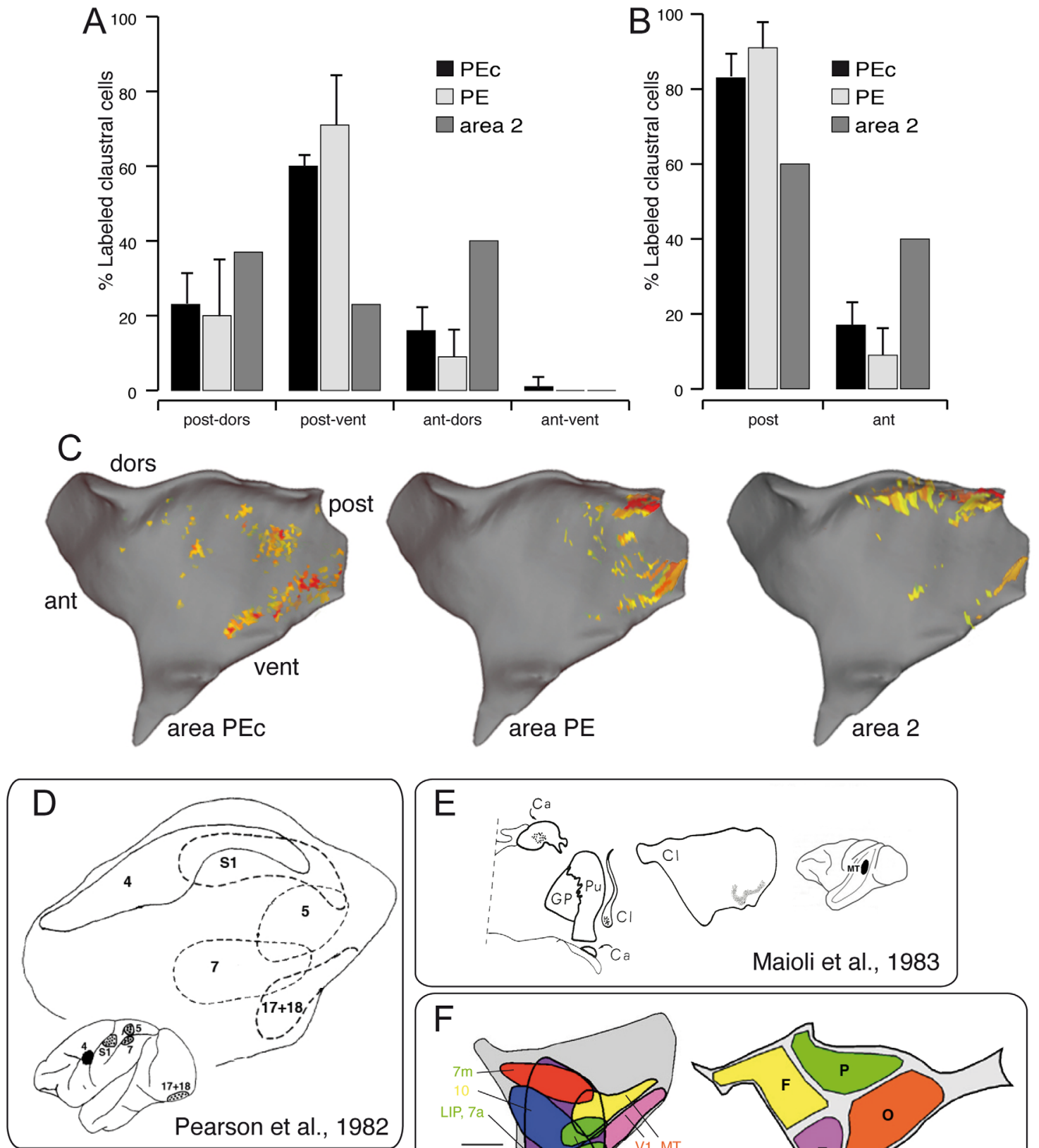

Maioli et al., 1983

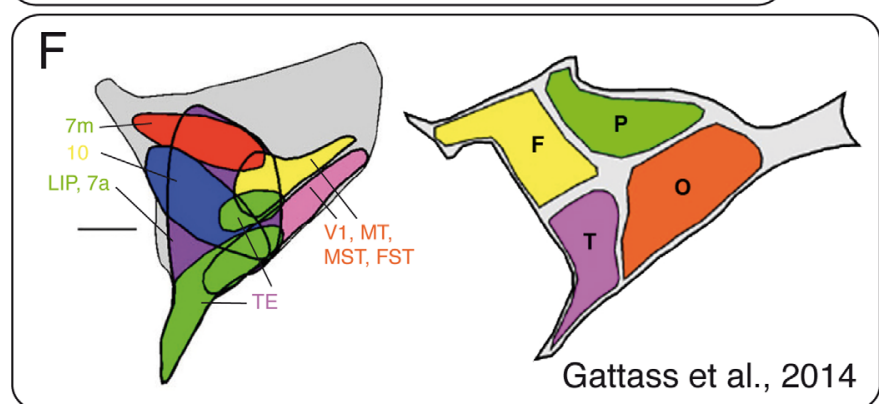

Figure 10. Summary and comparison with previous studies. A: Percentages of labeled cells in different quadrants of the claustrum after tracer injections in area PEc (three cases, mean \pm SD), PE (four cases, mean \pm SD), and area 2 (one case). B: Graph highlighting the predominance of label in the posterior part of the claustrum following injections in areas PE and PEc, and the more balanced distribution following injection in area 2. C: Average distributions of labeled cells following injections in the three areas, following morphing of individual reconstructions to a standard representation of the claustrum (based on case 2 of the present sample). Although there is wide overlap between the distributions of cells projecting to different targets, there is a gradual shift from ventral, to posterior, to dorsal locations as one considers the results of injections in areas PEc, PE and 2. D-F: Comparable lateral reconstructions of the claustrum modified from previous studies, shown in comparable orientation. Scale bar $=4 \mathrm{~mm}$ in $\mathrm{F}$. 
than conforming to some simple topographic rule, these neurons form multiple patches of origin separated by significant gaps, across relatively large territories of the claustrum.

Because the claustrum appears to be connected to the whole cortex, Crick and Koch (2005) hypothesized that it is the structure where sensory information is bound, functioning as a generator of the unified perception of a multitude of sensory stimuli. According to this view, the role of the claustrum would be important to rapidly integrate and bind information between neurons that are located across distinct cortical and thalamic regions. The present results appear to be compatible with this view. The spatially diffuse nature of the claustrocortical projections also seems compatible with the proposal recently advanced by Reser and coworkers (2014) that the claustrum promotes the "switch" between different cortical networks, as the "default" resting state network and task-specific networks. In both these cases, specific cortical areas with distinct functional properties and architectural characteristics would need to receive afferents from large, overlapping portions of claustrum to allow recombination and redistribution of information according to behavioral demands.

\section{ACKNOWLEDGMENTS}

The authors thank M. Verdosci, F. Campisi, and G. Placenti for technical assistance.

\section{CONFLICT OF INTEREST STATEMENT}

The authors declare that they have no conflicts of interest.

\section{ROLE OF AUTHORS}

All authors had full access to all the data in the study and take responsibility for the integrity of the data and the accuracy of the data analysis. Study concept and design: MG, CG, PF. Acquisition of data: MG, LP, SB, DI, MR. Analysis and interpretation of data: MG, CG. Drafting of the manuscript: MG, CG, PF, MR. Critical revision of the manuscript for important intellectual content: MR, LP, SB. Obtained funding: CG, PF, SB, MR. Administrative, technical, and material support: LP, DI. Study supervision: MG, CG, PF.

\section{LITERATURE CITED}

Baizer JS, Desimone R, Ungerleider LG. 1993. Comparison of subcortical connections of inferior temporal and posterior parietal cortex in monkeys. Vis Neurosci 10:59-72.

Baizer JS, Sherwood CC, Noonan M, Hof PR. 2014. Comparative organization of the claustrum: what does structure tell us about function? Front Syst Neurosci 8:117.
Bakola S, Gamberini M, Passarelli L, Fattori P, Galletti C. 2010. Cortical connections of parietal field PEc in the macaque: linking vision and somatic sensation for the control of limb action. Cereb Cortex 20:2592-2604.

Bakola S, Passarelli L, Gamberini M, Fattori P, Galletti C. 2013. Cortical connectivity suggests a role in limb coordination for macaque area PE of the superior parietal cortex. J Neurosci 33:6648-6658.

Batista AP, Buneo CA, Snyder LH, Andersen RA. 1999. Reach plans in eye-centered coordinates. Science 285:257-260.

Battaglia-Mayer A, Ferraina S, Genovesio A, Marconi B, Squatrito S, Molinari M, Lacquaniti F, Caminiti R. 2001. Eye-hand coordination during reaching. II. An analysis of the relationships between visuomanual signals in parietal cortex and parieto-frontal association projections. Cereb Cortex 11:528-544.

Bremner LR, Andersen RA. 2012. Coding of the reach vector in parietal area $5 \mathrm{~d}$. Neuron 75:342-351.

Breveglieri R, Galletti C, Gamberini M, Passarelli L, Fattori P. 2006. Somatosensory cells in area PEc of macaque posterior parietal cortex. J Neurosci 26:3679-3684. Erratum in: J Neurosci 2006;26:4452.

Breveglieri R, Galletti C, Monaco S, Fattori P. 2008. Visual, somatosensory, and bimodal activities in the macaque parietal area PEc. Cereb Cortex 18:806-816.

Brodmann K. 1909. Vergleichende Localisationslehre der Grosshirnrinde in Ihren Prinzipien Dargestellt auf Grund des Zellenbaues. Leipzig: Barth JA.

Burbaud P, Doegle C, Gross C, Bioulac B. 1991. A quantitative study of neuronal discharge in areas 5,2 , and 4 of the monkey during fast arm movements. J Neurophysiol 66: 429-443.

Burman KJ, Reser DH, Richardson KE, Gaulke H, Worthy KH, Rosa MGP. 2011. Subcortical projections to the frontal pole in the marmoset monkey. Eur J Neurosci 34:303-319.

Carman JB, Cowan WM, Powell TP. 1964. The cortical projection upon the claustrum. J Neurol Neurosurg Psychiatry 27:46-51.

Chadzypanagiotis D, Narkiewicz O. 1971. Connections of the visual cortex with the claustrum. Acta Neurobiol Exp (Wars) 31:291-311.

Crick FC, Koch C. 2005. What is the function of the claustrum? Philos Trans R Soc Lond B Biol Sci 360:12711279.

Druga R. 1968. Cortico-claustral connections. II. Connections from the parietal, temporal and occipital cortex to the claustrum. Folia Morphol (Praha) 16:142-149.

Duffy FH, Burchfiel JL. 1971. Somatosensory system: organizational hierarchy from single units in monkey area 5 . Science 172:273-275.

Ettlinger G, Wilson WA. 1990. Cross-modal performance: behavioural processes, phylogenetic considerations and neural mechanisms. Behav Brain Res 40:169-192.

Ferraina S, Bianchi L. 1994. Posterior parietal cortex: functional properties of neurons in area 5 during an instructed-delay reaching task within different parts of space. Exp Brain Res 99:175-178.

Ferraina S, Battaglia-Mayer A, Genovesio A, Marconi B, Onorati P, Caminiti R. 2001. Early coding of visuomanual coordination during reaching in parietal area PEC. J Neurophysiol 85:462-467.

Galletti C, Gamberini M, Kutz DF, Fattori P, Luppino G, Matelli M. 2001. The cortical connections of area V6: an occipito-parietal network processing visual information. Eur J Neurosci 13:1572-1588.

Galletti C, Gamberini M, Kutz DF, Baldinotti I, Fattori P. 2005. The relationship between $\mathrm{V} 6$ and $\mathrm{PO}$ in macaque extrastriate cortex. Eur J Neurosci 21:959-970. 
Gallyas F. 1979. Silver staining of myelin by means of physical development. Neurol Res 1:203-209.

Gamberini M, Passarelli L, Fattori P, Zucchelli M, Bakola S, Luppino G, Galletti C. 2009. Cortical connections of the visuomotor parietooccipital area V6Ad of the macaque monkey. J Comp Neurol 513:622-642.

Gattass R, Soares JG, Desimone R, Ungerleider LG. 2014. Connectional subdivision of the claustrum: two visuotopic subdivisions in the macaque. Front Syst Neurosci 8:63.

Hadjidimitrakis K, Dal Bo' G, Breveglieri R, Galletti C, Fattori P. 2015. Overlapping representations for reach depth and direction in caudal superior parietal lobule of macaques. J Neurophysiol 114:2340-2352.

Kalaska JF. 1996. Parietal cortex area 5 and visuomotor behavior. Can J Physiol Pharmacol 74:483-498.

Kemp JM, Powell TP. 1970. The cortico-striate projection in the monkey. Brain 93:525-546.

Lacquaniti F, Guigon E, Bianchi L, Ferraina S, Caminiti R. 1995. Representing spatial information for limb movement: role of area 5 in the monkey. Cereb Cortex 5: 391-409.

Luppino G, Hamed SB, Gamberini M, Matelli M, Galletti C. 2005. Occipital (V6) and parietal (V6A) areas in the anterior wall of the parieto-occipital sulcus of the macaque: a cytoarchitectonic study. Eur J Neurosci 21:3056-3076.

Maioli MG, Squatrito S, Battaglini PP, Rossi R, Galletti C. 1983. Projections from the visual cortical region of the superior temporal sulcus to the striatum and claustrum in the macaque monkey. Arch Ital Biol 121:259-266.

Mathur BN. 2014. The claustrum in review. Front Syst Neurosci 8:48.

Mesulam MM, Rosene DL. 1979. Sensitivity in horseradish peroxidase neurohistochemistry: a comparative and quantitative study of nine methods. J Histochem Cytochem 27:763-773.

Milardi D, Bramanti P, Milazzo C, Finocchio G, Arrigo A, Santoro G, Trimarchi F, Quartarone A, Anastasi G, Gaeta M. 2015. Cortical and subcortical connections of the human claustrum revealed in vivo by constrained spherical deconvolution tractography. Cereb Cortex 25:406414.

Minciacchi D, Granato A, Barbaresi P. 1991. Organization of claustro-cortical projections to the primary somatosensory area of primates. Brain Res 553:309-312.

Mountcastle VB, Lynch JC, Georgopoulos A, Sakata H, Acuña C. 1975. Posterior parietal association cortex of the monkey: command function for operations within extrapersonal space. J Neurophysiol 38:871-908.

Olson CR, Graybiel AM. 1980. Sensory maps in the claustrum of the cat. Nature 288:479-481.

Padberg J, Franca JG, Cooke DF, Soares JG, Rosa MG, Fiorani Jr. M, Gattass R, Krubitzer L. 2007. Parallel evolution of cortical areas involved in skilled hand use. J Neurosci 27:10106-10115.

Pandya DN, Seltzer B. 1982. Intrinsic connections and architectonics of posterior parietal cortex in the rhesus monkey. J Comp Neurol 204:196-210.
Passarelli L, Rosa MG, Gamberini M, Bakola S, Burman KJ, Fattori P, Galletti C. 2011. Cortical connections of area V6Av in the macaque: a visual-input node to the eye/ hand coordination system. J Neurosci 31:1790-17801.

Pearson RC, Brodal P, Gatter KC, Powell TP. 1982. The organization of the connections between the cortex and the claustrum in the monkey. Brain Res 234:435-441.

Piserchia V, Breveglieri R, Hadjidimitrakis K, Bertozzi F, Galletti C, Fattori P. 2016. Mixed body/hand reference frame for reaching in $3 D$ space in macaque parietal area PEc. Cereb Cortex. Mar 3:pii: bhw039.

Pons TP, Kaas JH. 1986. Corticocortical connections of area 2 of somatosensory cortex in macaque monkeys: a correlative anatomical and electrophysiological study. J Comp Neurol 248:313-335.

Reser DH, Burman KJ, Yu HH, Chaplin TA, Richardson KE, Worthy KH, Rosa MG. 2013. Contrasting patterns of cortical input to architectural subdivisions of the area $8 \mathrm{com}$ plex: a retrograde tracing study in marmoset monkeys. Cereb Cortex 23:1901-1922.

Reser DH, Richardson KE, Montibeller MO, Zhao S, Chan JM, Soares JG, Chaplin TA, Gattass R, Rosa MG. 2014. Claustrum projections to prefrontal cortex in the capuchin monkey (Cebus apella). Front Syst Neurosci 8:123.

Rosa MG, Tweedale R. 2005. Brain maps, great and small: lessons from comparative studies of primate visual cortical organization. Philos Trans R Soc Lond B Biol Sci 360: 665-691.

Rosa MG, Palmer SM, Gamberini M, Burman KJ, Yu HH, Reser DH, Bourne JA, Tweedale R, Galletti C. 2009. Connections of the dorsomedial visual area: pathways for early integration of dorsal and ventral streams in extrastriate cortex. J Neurosci 29:4548-4563.

Sakata H, Takaoka Y, Kawarasaki A, Shibutani H. 1973. Somatosensory properties of neurons in superior parietal cortex (area 5) of the rhesus monkey. Brain Res 64:85-102.

Shima K, Hoshi E, Tanji J. 1996. Neuronal activity in the claustrum of the monkey during performance of multiple movements. J Neurophysiol 76:2115-2119.

Smythies J, Edelstein L, Ramachandran V. 2012. Hypotheses relating to the function of the claustrum. Front Integr Neurosci. 6:53.

Tanné-Gariepy J, Boussaoud D, Rouiller EM. 2002. Projections of the claustrum to the primary motor, premotor, and prefrontal cortices in the macaque monkey. J Comp Neurol 454:140-157.

Taoka M, Toda T, Iwamura Y. 1998. Representation of the midline trunk, bilateral arms, and shoulders in the monkey postcentral somatosensory cortex. Exp Brain Res 123:315-22.

Taoka M, Toda T, Iriki A, Tanaka M, Iwamura Y. 2000. Bilateral receptive field neurons in the hindlimb region of the postcentral somatosensory cortex in awake macaque monkeys. Exp Brain Res 134:139-146.

Van Essen DC, Drury HA, Dickson J, Harwell J, Hanlon D, Anderson $\mathrm{CH}$. 2001. An integrated software suite for surface-based analyses of cerebral cortex. J Am Med Inform Assoc 8:443-459. 